\section{Evaluación del cuestionario SF-12: verificación de la utilidad de la escala salud mental}

\author{
PABLO VERA-VILLARROEL ${ }^{1, \mathrm{a}}$, JAIME SILVA ${ }^{2, \mathrm{a}}$, \\ KAREM CELIS-ATENAS ${ }^{1,3, a}$, PAULA PAVEZ ${ }^{1, b}$
}

\section{Evaluation of the SF-12. Usefulness of the mental health scale}

\begin{abstract}
Background: The SF-12 is one of the most used questionnaires to evaluate the multidimensional health related quality of life, worldwide. However, the factor structure does not match the structure of the original instrument. Besides, most studies centered in the physical dimension paying less attention to mental health related quality of life. Aim: To evaluate the psychometric properties of the SF-12 and to validate the mental health dimension of this questionnaire. Material and Methods: Two studies were conducted, the first consisting of 840 people aged between 18 and 60 years, who answered the SF-12. In the second study, 970 people aged between 18 and 60 years answered the SF-12 questionnaire, the Beck Depression Inventory (BDI) and the State-Trait Anxiety Inventory (STAI). Reliability (internal consistency and temporal stability) was analyzed, along with construct and criterion validity for the mental health dimension. Results: The reliability estimate using Cronbach's alpha in both studies was higher than 0.74 in both dimensions (physical and psychological). Construct validity analysis yielded a three factor complex solution. Finally the criterion validity analysis showed appropriate and significant correlations with depression and anxiety. Conclusions: The SF-12 and its mental health scale are suitable for epidemiological characterization studies.
\end{abstract}

(Rev Med Chile 2014; 142: 1275-1283)

Key words: Mental health, Quality of life; Questionnaires.

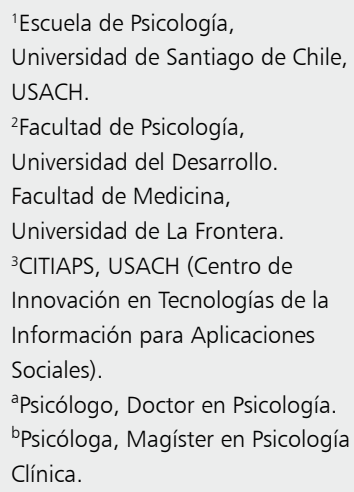

Esta investigación contó con el apoyo del proyecto FONDECYT № 1110520, Universidad de Santiago de Chile, USACH. Agradecimiento a proyecto USA 1204 del Ministerio de Educación/CITIAPS.

Recibido el 30 de enero de 2013, aceptado el 27 de septiembre de 2014.

Correspondencia a:

Dr. Pablo Vera-Villarroel Escuela de Psicología. Universidad de Santiago de Chile, USACH. Avenida Ecuador $3650,3^{\circ}$ Piso. Santiago.

pablo.vera@usach.cl.

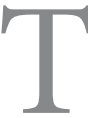
radicionalmente la evaluación en salud ha estado basada en indicadores objetivos (ej. bioquímicos, fisiológicos y anatómicos). Sin embargo, el enfoque moderno de salud que deriva del concepto de completo bienestar físico, mental y social propuesto por la Organización Mundial de la Salud, ya no solamente está centrado en la ausencia de infecciones o enfermedades, sino que pone de relieve la necesidad de incluir evaluaciones tanto objetivas como subjetivas para esta variable ${ }^{1-3}$, así como en la valoración de los recursos psicológicos que favorecen la salud y el bienestar $^{4-9}$.

La actual estimación de salud incluye medidas subjetivas como la calidad de vida relacionada con la salud $(\mathrm{CVRS})^{10}$. Esta variable es usualmente utilizada tanto en población general, como en la con alguna patología específica, porque permite obtener un perfil acabado del paciente e $^{1-3,11-17}$.

Como medida más ampliamente utilizada para esta valoración, se encuentra la familia de los cuestionarios $\mathrm{SF}^{3}$, cuyo propósito es estimar el estado funcional del paciente, reflejando su salud física, mental y social, así como la obtención de un valor cuantitativo, que representará el estado de salud de forma multidimensional ${ }^{1,18}$.

La versión original de los SF consta de treinta y seis ítems (SF-36) desarrollados y utilizados en 
la década 1990-99 en investigaciones de corte epidemiológico ${ }^{2,3}$, por el hecho de proporcionar un perfil del estado de salud percibido, que valora tanto estados positivos como negativos en población con y sin patología $a^{19-23}$. Esto hace de este instrumento uno de los más ampliamente utilizados en el mundo, por relacionar salud con calidad de vida ${ }^{17-29}$.

El SF incluye dos dimensiones que miden ocho conceptos de salud, como la función física (grado que la salud limita las actividades físicas), función social (grado de salud física y emocional que afectan la vida social habitual), rol físico (grado donde la salud física interfiere en el trabajo y las actividades diarias), rol emocional (grado en que los problemas emocionales interfieren en el trabajo $\mathrm{u}$ otras actividades diarias), salud mental (salud mental general), vitalidad (sentimiento de energía y vitalidad), dolor corporal (intensidad del dolor) y salud general (valoración personal de la salud) ${ }^{1,3}$.

Estas ocho escalas forman dos grupos distintos relacionados con la salud física y mental de las personas. No obstante, autores como Gandek ${ }^{29}$, han propuesto la necesidad de desarrollar un nuevo cuestionario que evalúe estas dos dimensiones globales, pero con menos ítems. Un ejemplo de esto es el SF-12 $2^{31}$, caracterizado por ser una versión abreviada del cuestionario original con sólo doce ítems (tiempo de aplicación de dos minutos aproximadamente), cuyo objetivo es evaluar el grado de bienestar y capacidad funcional de las personas mayores de 14 años $^{2-3}$.

Las investigaciones que utilizan estos doce ítems del SF han verificado que el instrumento es una medida válida y confiable ${ }^{25,27,32}$, tanto en la escala original como en sus distintas versiones ( 12 ítems, 8 ítems o 6 ítems $)^{1,13}$. Sin embargo, a pesar de estos resultados, los estudios se han centrado principalmente en la evaluación de la dimensión física relacionada con la calidad de vida, existiendo escasez de investigaciones que incorporan la dimensión salud mental ${ }^{33-36}$. Además de esta dificultad, es importante apreciar que algunas investigaciones han encontrado que, aunque este instrumento ha mostrado una aceptable validez y fiabilidad, en algunos países y culturas, no ha coincidido con la estructura prevista del instrumento original ${ }^{32,37}$. Esto hace necesario contar con estudios comparativos del estado de salud actualizados para diversos países, que permitan obtener adaptaciones culturales que contribuyan a la estandarización de los resultados y entreguen herramientas fiables para la planificación en salud ${ }^{1}$.

Ambas necesidades son las que guían el objetivo de esta investigación, es decir, evaluar las propiedades métricas del cuestionario (SF-12) y validar la dimensión salud mental de este cuestionario. Para ello, se realizaron dos estudios: en el primero, el objetivo fue estimar la confiabilidad (consistencia interna y estabilidad temporal) y análisis factorial exploratorio, mientras que el segundo tuvo como objetivo, evaluar consistencia interna, validez de constructo y validez de criterio con variables psicológicas.

\section{Estudio 1}

\section{Objetivo}

Evaluar las propiedades métricas del cuestionario SF-12.

\section{Participantes y Método}

Participaron 840 personas en esta investigación (394 hombres y 446 mujeres) entre 18 y 60 años con una media de 33,44 años $(\mathrm{DE}=12,17)$, la mayor proporción de la muestra cursó estudios secundarios completos y reportan pertenecer principalmente en el nivel socioeconómico medio. La selección de la muestra fue intencionada, con participación voluntaria. No se entregó ningún tipo de gratificación, debiendo cada sujeto firmar consentimiento escrito. Como criterio de exclusión, se descartó a personas con patología mental diagnosticada. Hubo una mortalidad aproximada de $10 \%$ de los cuestionarios repartidos.

\section{Instrumentos}

Cuestionario $\mathrm{SF}-12^{27}$. Cuestionario de calidad de vida relacionada con la salud. Compuesto por doce ítems, cuya finalidad es otorgar un instrumento de fácil aplicación para evaluar el grado de bienestar y capacidad funcional de las personas mayores de 14 años $^{1-3}$, definiendo un estado positivo y negativo de la salud física y mental, por medio de ocho dimensiones (función física, rol físico, dolor corporal, salud mental, salud general, vitalidad, función social y rol emocional), algunos ítems del instrumento son: "En general, usted diría que su salud es", "Durante las 4 últimas semanas, ¿Hasta qué punto su salud física o los problemas emocionales han dificultado sus actividades socia- 
les habituales con la familia, los amigos/as, los vecinos/as u otras personas?", " $i$ Tuvo dolor en alguna parte del cuerpo durante las 4 últimas semanas?". Las opciones de respuesta forman escalas de tipo Likert (donde el número de opciones varía de tres a seis puntos, dependiendo del ítem), que evalúan intensidad y/o frecuencia del estado de salud de las personas. El puntaje va entre 0 y 100 , donde el mayor puntaje implica una mejor calidad de vida relacionada con la salud. Las investigaciones que utilizan los doce ítems del SF, han verificado que este instrumento es una medida válida y confiable, encontrándose estimaciones de consistencia interna superiores a 0,70 y correlaciones significativas entre las versiones de la escala ${ }^{18,25,27,51}$.

\section{Procedimiento}

Encuestadores entrenados para esta tarea tomaron contacto en diversos espacios públicos y privados (universidades, instituciones, empresas y vía pública), con el fin de realizar la aplicación de los cuestionarios. A cada persona que aceptó participar, se le explicó la tarea a realizar y se le hizo entrega del conjunto de cuestionarios, previa lectura y firma del consentimiento informado, este fue aprobado por los comités de ética de las universidades patrocinantes. Una vez finalizada la aplicación, se digitó en una base de datos usando el programa estadístico SPSS versión 19, para el posterior análisis métrico de los datos.

Para llevar a cabo el objetivo de este primer estudio, se estimaron los niveles de confiabilidad (consistencia interna y estabilidad temporal) y validez de constructo. La estimación de la confiabilidad se realizó a través del análisis de consistencia interna, con la técnica de alfa de Cronbach, mientras que la validez de constructo, se realizó mediante el análisis factorial exploratorio. Cada uno de los cálculos se llevó a cabo para el total de la muestra.

A fin de evaluar la estabilidad temporal del total de los participantes, se extrajo una muestra aleatoria de 180 sujetos, a los cuales pasadas tres semanas, se les aplicó nuevamente el instrumento. De estos, 105 fueron mujeres y 75 hombres con edades entre 18 y 60 años.

\section{Resultados}

En Tabla 1 se presentan los estadísticos descriptivos, de variabilidad y consistencia interna para cada una de las dimensiones del SF-12. La evaluación de consistencia interna tanto para la dimensión física como mental, arroja adecuados indicadores.

\section{Estimación de confiabilidad}

La evaluación de la consistencia interna (alfa de Cronbach) para la dimensión física fue de 0,63 y para la dimensión mental fue de 0,72 . La correlación dominio/dominio fue significativa, encontrándose correlaciones adecuadas para ambas dimensiones, a saber, para la dimensión mental, una correlación de 0,33 y para la dimensión física 0,50 , indicando la estabilidad de ambas dimensiones ${ }^{47}$.

\section{Validez de constructo}

Para la validez de constructo, se ejecutó un análisis factorial exploratorio, por medio de componentes principales con rotación Varimax. Los indicadores de ajuste de la matriz de datos fueron significativos con medida de adecuación muestral de Kaiser Meyer Olkin de 0,83 y Prueba de esfericidad de Bartlett $\left(\chi_{(66)}^{2}=3218,822 ; \mathrm{p}=0,000\right)$.

Del análisis de componentes principales con rotación Varimax, resultó una solución factorial compleja de la cual se interpretan tres dimensiones dominantes latentes con pesos factoriales en más de un factor, que explican 58,36\% de la varianza. El primer factor que explicó 36,30\% contiene a los ítems de las dimensiones salud mental, vitalidad, función social y salud general; el segundo factor que explicó $11,78 \%$ contiene los ítems de las dimensiones rol físico y rol emocional, y finalmente el tercer factor que explicó $10,29 \%$ contiene a los

\section{Tabla 1. Estadísticos descriptivos, variabilidad y consistencia interna para cada una de las dimen- siones de cuestionario SF-12}

\begin{tabular}{|lccc|}
\hline & Media & $\begin{array}{c}\text { Total } \\
\text { DE }\end{array}$ & $\boldsymbol{\alpha}$ \\
Dimensión física & 80,25 & 15,77 & 0,74 \\
Función física & 88,65 & 21,47 & 0,68 \\
Rol físico & 82,60 & 21,84 & 0,79 \\
Dolor corporal & 80,63 & 24,77 & \\
Salud general & 58,76 & 21,45 & \\
Dimensión mental & 77,42 & 17,10 & 0,80 \\
Vitalidad & 70,92 & 24,62 & \\
Función social & 82,58 & 22,18 & \\
Rol emocional & 83,66 & 21,49 & 0,84 \\
Salud mental & 83,66 & 21,49 & 0,57 \\
\hline
\end{tabular}


ítems de función física y dolor corporal, cada uno de los factores se compone con aquellos ítems con mayor carga factorial, en caso de existir cargas en más de un factor y con carga significativas $(\geq 0,30)^{48}$ (Tabla 2).

\section{Estudio 2}

\section{Objetivo}

Validar la dimensión salud mental del cuestionario SF-12.

\section{Participantes y Método}

En este segundo estudio participaron 970 personas (442 hombres y 528 mujeres) entre 18 y 60 años con una media de 33,45 años $(\mathrm{DE}=12,16)$. La mayor proporción de la muestra cursó estudios secundarios completos y reportan pertenecer principalmente al nivel socioeconómico medio. La selección de la muestra fue intencionada, con participación voluntaria, como criterio de exclusión, se descartó a personas con patología mental diagnosticada. No se entregó ningún tipo de gratificación, cada participante firmó el consentimiento informado. Hubo una mortalidad aproximada de $8 \%$ de los cuestionarios repartidos.

\section{Instrumentos}

Inventario de Depresión de Beck (BDI, revised version $)^{38}$. Este inventario mide gravedad e intensidad de los síntomas cognitivos, afectivos, conductuales y psicofisiológicos de la depresión por medio de veintiún reactivos ${ }^{38}$. El rango de la puntuación obtenida es de 0 a 63 puntos. Se observan elevados índices de consistencia interna tanto en la validación española del cuestionario 0,83 y $0,90^{39-42}$, como en diversos estudios internacionales ${ }^{39,43,58-60}$.

Inventario de Ansiedad Estado-Rasgo ${ }^{44}$. El test, en su forma Ansiedad Rasgo (A-R), evalúa la ansiedad como una condición emocional permanente de tensión, y su forma Ansiedad Estado (A-E), tiene por objetivo medir la ansiedad o condición emocional transitoria del organismo humano ${ }^{44}$. La adaptación española presenta adecuados indicadores de confiabilidad y valide $z^{45-46}$ al igual que en la adaptación chilena, donde se obtiene un alfa de Cronbach de 0,92 para la escala estado y de 0,87 para la escala rasgo, mientras que la estructura factorial, está acorde a lo mostrado por otras investigaciones ${ }^{47}$.

\section{Tabla 2. Matriz de componentes principales con rotación Varimax y alfa de Cronbach para cada dimisión obtenida}

\begin{tabular}{|lccc|}
\hline & Factor 1 & $\begin{array}{c}\text { Total } \\
\text { Factor 2 }\end{array}$ & Factor 3 \\
\hline Ítem 9d & 0,711 & & \\
Ítem 9f & 0,708 & & \\
Ítem 9e & 0,664 & & \\
Ítem 6 & 0,535 & 0,388 & \\
Ítem 1 & 0,518 & & 0,474 \\
Ítem 4c & & 0,781 & \\
Ítem 4b & 0,758 & 0,320 \\
Ítem 5c & 0,483 & 0,660 & \\
Ítem 5b & 0,526 & 0,645 & \\
Ítem 3d & & & 0,794 \\
Ítem 3b & & & 0,760 \\
Ítem 8 & & & 0,532 \\
\hline Alfa de cronbach & 0,73 & 0,79 & 0,65 \\
\hline
\end{tabular}

Los ítems son ordenados en base a cargas factoriales descendentes dominantes $(>0=0,30)$.

\section{Procedimiento}

Un grupo de encuestadores entrenados para esta tarea tomó contacto en diversos espacios públicos y privados (universidades, instituciones, empresas y vía pública) con el fin de realizar la aplicación de los cuestionarios. A cada persona que aceptó participar, se le explicó la tarea a realizar y se le hizo entrega del conjunto de cuestionarios, previa lectura y firma del consentimiento informado, que fue aprobado por los comités de ética de las universidades patrocinantes. Una vez finalizada la aplicación de cuestionarios, se digitó en una base de datos usando el programa estadístico SPSS versión 19, para el posterior análisis métrico de los datos.

Para validar la dimensión salud mental del cuestionario SF-12, fue necesaria la estimación de la confiabilidad, por medio de consistencia interna a través del cálculo de alfa de cronbach, validez de constructo y criterio.

La evaluación de la validez de constructo, se realizó mediante el análisis factorial exploratorio, mientras que la estimación de validez de criterio se llevó a cabo correlacionando las dimensiones definidas teóricamente del cuestionario SF-12 (dimensión metal y física), ya que la evidencia empírica indicaría que estas dimensiones corre- 
lacionarían de formas inversa con el cuestionario de depresión de Beck (BDI) y el cuestionario de Ansiedad Estado Rasgo (STAI) ${ }^{48,49}$.

\section{Resultados}

En Tabla 3 se presentan los estadísticos descriptivos, de variabilidad y consistencia interna para cada una de las dimensiones del SF-12. La evaluación de consistencia interna, tanto para la dimensión física como mental, entrega adecuados indicadores.

\section{Validez de constructo}

Respecto a la validez de constructo se ejecutó un análisis factorial exploratorio, por medio de componentes principales con rotación Varimax. Los indicadores de ajuste de la matriz de datos fueron significativos con medida de adecuación muestral de Kaiser Meyer Olkin de 0,846 y Prueba de esfericidad de Bartlett $\left(\chi_{(66)}^{2}=4117,222\right.$; $\mathrm{p}=0,000)$.

Del análisis de componentes principales con rotación Varimax, resultó una solución compleja de tres factores que explicaron $60,31 \%$ de la varianza, encontrándose ítems con carga en más de un factor. El primer factor que explicó 38,38\% contiene a los ítems de las dimensiones salud mental, vitalidad, función social y salud general; el segundo factor que explicó $11,78 \%$ contiene los ítems de las dimensiones rol físico y mental, y finalmente el tercer factor que explicó $10,15 \%$ contiene los ítems de función física y dolor corporal.

Tabla 3. Estadísticos descriptivos, variabilidad y consistencia interna para cada una de las dimensiones de cuestionario SF-12

\begin{tabular}{|lccc|}
\hline & Media & $\begin{array}{c}\text { Total } \\
\text { DE }\end{array}$ & $\boldsymbol{\alpha}$ \\
\hline Dimensión física & 82,04 & 22,49 & 0,75 \\
Función física & 89,09 & 21,08 & 0,69 \\
Rol físico & 82,04 & 22,49 & 0,81 \\
Dolor corporal & 80,73 & 25,35 & \\
Salud general & 58,57 & 22,04 & \\
Dimensión mental & 77,40 & 17,90 & 0,82 \\
Vitalidad & 70,54 & 24,82 & \\
Función social & 82,87 & 22,40 & \\
Rol emocional & 83,57 & 22,32 & 0,84 \\
Salud mental & 83,57 & 22,32 & 0,59 \\
\hline
\end{tabular}

La selección de ítems para cada factor es en base a mayor carga factorial y con carga significativas $(\geq 0,30)^{48}$ (Tabla 4$)$.

\section{Validez de criterio}

Se evalúo la validez de criterio por medio de la correlación de las dimensiones del cuestionario SF-12 con el cuestionario de depresión de Beck (BDI) y el cuestionario de ansiedad estado rasgo (STAI). Todas las correlaciones obtenidas son significativas, adecuadas y negativas (Tabla 5).

\section{Tabla 4. Matriz de componentes principales con rotación Varimax y alfa de Cronbach para cada dimensión obtenida}

\begin{tabular}{|lccc|}
\hline & \multicolumn{1}{|c|}{$\begin{array}{c}\text { Total } \\
\text { Factor 1 }\end{array}$} & Factor 2 & Factor 3 \\
\hline Ítem 9d & 0,735 & & \\
Ítem 9e & 0,722 & & \\
Ítem 9f & 0,702 & & \\
Ítem 6 & 0,546 & 0,330 & 0.318 \\
Ítem 1 & 0,461 & & 0,455 \\
Ítem 4c & & 0,814 & \\
Ítem 4b & & 0,794 & \\
Ítem 5c & 0,467 & 0,669 & \\
Ítem 5b & 0,552 & 0,635 & \\
Ítem 3d & & & 0,810 \\
Ítem 3b & & & 0,776 \\
\hline Ítem 8 & 0,301 & & 0,590 \\
\hline Alfa de Cronbach & 0,75 & 0,81 & 0,68 \\
\hline
\end{tabular}

Los ítems son ordenados en base a cargas factoriales descendentes dominantes $(>0=0,30)$.

Tabla 5. Correlación entre SF-12, BDI y STAI

\begin{tabular}{|c|c|c|c|}
\hline & BDI & $\begin{array}{l}\text { STAI- } \\
\text { Estado }\end{array}$ & $\begin{array}{c}\text { STAI- } \\
\text { Rasgo }\end{array}$ \\
\hline 1. Dimensión física & $-0,42 * *$ & $-0,39 * *$ & $-0,44 * *$ \\
\hline 2. Función física & $-0,30 * *$ & $-0,26 * *$ & $-0,29 * *$ \\
\hline 3. Rol físico & $-0,24 * *$ & $-0,24^{* *}$ & $-0,28 * *$ \\
\hline 4. Dolor corporal & $-0,41 * *$ & $-0,36^{* *}$ & $-0,40 * *$ \\
\hline 5. Salud general & $-0,32 * *$ & $-0,33^{* *}$ & $-0,34 * *$ \\
\hline 6. Dimensión mental & $-0,56^{* *}$ & $-0,56^{* *}$ & $-0,60 * *$ \\
\hline 7. Vitalidad & $-0,36 * *$ & $-0,38^{* *}$ & $-0,40 * *$ \\
\hline 8. Función social & $-0,50 * *$ & $-0,44 * *$ & $-0,48 * *$ \\
\hline 9. Rol emocional & $-0,43^{* *}$ & $-0,38^{* *}$ & $-0,44 * *$ \\
\hline 10. Salud mental & $-0,43^{* *}$ & $-0,38^{* *}$ & $-0,44^{* *}$ \\
\hline
\end{tabular}




\section{Discusión}

El objetivo de este estudio fue caracterizar las propiedades métricas del cuestionario SF-12 y validar la dimensión salud mental de este cuestionario, con el fin de contar con una herramienta válida para la valoración de un perfil en salud, no sólo centrado en los aspectos físicos de la persona sino que en aquellos relacionados con la salud psicológica. Para ello se propusieron dos estudios, los cuales muestran adecuados indicadores de confiabilidad y validez.

La estimación de la consistencia interna tanto para la dimensión física como mental resultante es similar a las estimaciones en otros estudios , $32,50-51^{\text {. }}$ Además, si se considera lo sugerido por el Comité Científico asesor para resultados médicos confiables en el área de la evaluación de indicadores en calidad de vida, los valores de consistencia interna obtenidos para la dimensión física y mental, fueron adecuados para comparaciones grupales y en la evaluación de la estabilidad temporal ${ }^{52}$.

La adecuación y significación de las correlaciones obtenidas permitió inferir que tanto la dimensión física como mental mostraron estabilidad en el intervalo de tiempo evaluado (tres semanas) entre el test y re test, por lo tanto, se cuenta con una medida estable en el tiempo de la calidad de vida relacionada con la salud ${ }^{36,53}$.

Estudios empíricos de la estructura factorial del cuestionario proponen dos factores, dimensión física y mental ${ }^{51,52,55}$. Sin embargo, en distintos países y diversas culturas, no se ha podido verificar la misma estructura prevista del instrumento original $^{32,37}$. Lo obtenido en el presente estudio, evidenció una solución factorial compleja de tres factores, que pueden ser interpretados como una dimensión social, una mental/psíquica y una física. Lo anterior es coherente con la conceptualización del constructo de calidad de vida relacionado a la salud, entendida como un constructo multidimensional compuesto por un dominio físico, mental y social de la salud ${ }^{56}$.

Igualmente la validez de criterio indica que la dimensión salud mental del SF-12, es una medida útil para la evaluación de esta dimensión, en estudios generales con gran cantidad de población. Las correlaciones entre esta dimensión y las medidas de depresión y ansiedad (estado-rasgo), fueron adecuadas y negativas, en la línea de lo hipotetizado originalmente. De la misma forma lo encontrado es similar en cuanto al rango de correlación con muestras angloparlantes y española, tanto en población sana como en población clínica. Así por ejemplo, similares correlaciones se encuentran con la validación en una muestra griega $^{56}$, y en una muestra española de sujetos con enfermedades cardiacas $^{34}$, así también en muestra con daño neurológico y depresión ${ }^{51}$.

Los datos del presente estudio con población chilena muestra que el SF-12 es una escala con medidas válidas y fiables que permite la obtención de un perfil acabado del paciente que caracterice su estado de salud no sólo físico, sino que también mental y social, en relación a la calidad de vida relacionada con la salud ${ }^{57}$. Contando, por lo tanto, con una medida de fácil aplicación para uso individual, como para grandes poblaciones para los estados de salud psicológica que en otras condiciones requiere de mayor tiempo, y recursos.

Además al ser ésta una medida subjetiva, complementa la tradicional evaluación de salud, centrada solamente en indicadores objetivos, permitiendo valorar los efectos en la salud tanto física como mental de los tratamientos recibidos por los pacientes, en función de la percepción del individuo $^{3}$. Es de importancia para los ámbitos de salud el contar con herramientas psicométricas validas y confiables, estudios recientes han mostrado que ha pasado ha ser una prioridad en la investigación en salud ${ }^{61}$.

Futuras investigaciones podrían profundizar en la evaluación de la estructura factorial confirmatoria de este instrumento, dada la divergencia encontrada en investigaciones previas, considerando además factores relevantes como los culturales y la dimensión salud mental considerada en este estudio.

Se estima necesario contar con normas poblacionales que permitan identificar desviaciones en las puntuaciones de un individuo, en relación con lo esperado para su edad y sexo, para así evaluar el impacto de la enfermedad sobre la calidad de vida de las personas y establecer objetivos terapéuticos que relacionen salud física con la salud mental psicológica ${ }^{2,18,35}$.

\section{Referencias}

1. Monteagudo-Piqueras O, Hernando-Arizaleta L, Palomar-Rodríguez J. Normas poblacionales de referencia 
de la versión española del SF-12v2 para la Región de Murcia. Gac Sanit 2011; 25 (1): 50-61.

2. Rebollo P. Utilización del «grupo SF» de los cuestionarios de calidad de vida (SF-36, SF-12 y SF-6D) en el marco de ensayos clínicos y en la práctica clínica habitual. Med Clin (Barc) 2008; 130 (19): 740-1.

3. Tuesca-Molina R. La calidad de vida, su importancia y cómo medirla. Salud Uninorte. Barranquilla (Col.) 2005; 21: 76-86.

4. Vera-Villarroel P, Pavez P, Silva J. El rol predisponente del optimismo: Hacia un modelo etiológico del bienestar. Ter Psicol 2012; 30 (2): 74-84.

5. Chavarria MP, Barra E. Satisfacción vital en adolescentes: Relación con autoeficacia y el apoyo social percibido. Ter Psicol 2014; 32 (1): 41-6.

6. Park, N, Peterson, C, Sun, J. La psicología positiva: Investigación y Aplicaciones. Ter Psicol 2013; 31 (1): 11-9.

7. Vázquez C. Psicología positiva: Introducción al número especial. Ter Psicol 201331 (1): 5-10.

8. Moyano E, Cornejo F, Carreño M, Muñoz A. Bienestar Subjetivo en maximizadores y satisfactores. Ter Psicol 2013; 31 (3): 273-80.

9. de la Cruz-Sánchez E, Feu S, Vizuete-Carrizosa M. El nivel educativo como factor asociado al bienestar subjetivo y la salud mental en la población española. Univ Psychol 2013; 12 (1): 31-40.

10. Urzúa A, Caqueo-Urízar A. Calidad de vida: Una revisión teórica del concepto. Ter Psicol 2012; 30 (1): 61-72.

11. Chaves C, Vázquez C, Hervas G. Benefit finding and well being in children with threatening illnesses: An integrative. Ter Psicol 2013; 31 (1): 59-68.

12. Pickard AS, Wang Z, Walton SM, Lee TA. Are decisions using cost-utility analyses robust to choice of SF-36/ SF-12 preference-based algorithm? Health Qual Life Outcomes 2005; 3 (11): 11-20.

13. Vinaccia S, Quiceno JM. Calidad de vida relacionada con la salud y factores psicológicos: un estudio desde la enfermedad pulmonar obstructiva crónica-EPOC (Colombia). Ter Psicol 2011; 29 (1): 65-76.

14. Quinceno JM, Vinaccia S. Calidad de vida, factores salutogénicos e ideación suicida en adolescentes. Ter Psicol 2013; 31 (2): 263-71.

15. Vega V, Jenaro C, Flores M, Cruz M, Cerda A. Evaluación de la calidad de vida de adultos con discapacidad intelectual institucionalizados en Chile. Ter Psicol 2013; 12 (2): 471-81.

16. Urzúa A, Pavlov R, Cortés R, Pino V. Factores psicosociales relacionados con la calidad de vida en salud en pacientes hemodializados. Ter Psicol 2011; 29 (1): 13540.

17. Sánchez AI, Guglielmi O, Jurado-Gamez B, Buela-Casal
G. Efectos del síndrome de apneas-hipoapneas del sueño sobre la calidad de vida y la somnolencia diurna. Univ Psychol 2013; 12 (2): 601-11.

18. Vilagut G, Valderas JM, Ferrer M, Garín O, López-García E, Alonso J. Interpretación de los cuestionarios de salud SF-36 y SF-12 en España. Componentes físico y mental. Med Clin (Barc) 2008; 130 (19): 726-35.

19. Hann M, Reeves D. The SF-36 scales are not accurately summarised by independent physical and mental component scores. Qual Life Res 2008; 17 (3): 413-23.

20. Hawthorne G, Osborne R, Taylor A, Sansoni J. The SF36 version 2: Critical analyses of population weights, scoring algorithms and population norms. Qual Life Res 2007; 16 (4): 661-73.

21. Knox S, King M. Validation and calibration of the SF-36 health transition question against an external criterion of clinical change in health status. Qual Life Res 2009; 18 (5): 637-45.

22. Lugo L, García H, Gómez C. Confiabilidad del cuestionario de calidad de vida en salud SF-36 en Medellín, Colombia. Rev Fac Nac Salud Pública 2006; 24 (2): 37 50.

23. Wang W, López V, Sek Ying Ch, Thompson D. The psychometric properties of the Chinese version of the SF-36 health survey in patients with myocardial infarction in mainland China. Qual Life Res 2006; 15: 1525-31.

24. Aaronson NK, Muller M, Cohen PDA, Essink-Bot ML, Fekkes M, Sanderman R, et al. Translation, validation and norming of the Dutch language version of the SF36 Health Survey in community and chronic disease populations. J Clin Epidemiol 1998; 51 (11): 1055-68.

25. Andrews G. A brief integer scorer for the SF-12: Validity of the brief scorer in Australian community and clinic settings. Aus N Z J Public Health 2002; 26: 508-10.

26. Bullinger M. German translation and psychometric testing of the SF-36 Health Survey: preliminary results from the IQOLA Project. Soc Sci Med 1995; 41: 1359-66.

27. Jenkinson C, Layte R. Development and testing of the UK SF-12 (short form health survey). J Health Serv Res Policy 1997; 2: 14-8.

28. Lim LLY, Seubsman S, Sleigh A. The SF-36 health survey: Tests of data quality, scaling assumptions, reliability and validity in healthy men and women. Health Qual Life Outcomes 2008; 6: 52-60.

29. Taft C, Karlsson J, Sullivan M. Performance of the Swedish SF-36 version 2.0. Qual Life Res 2004; 13: 251-6.

30. Gandek B, Ware J, Aaronson N, Apolone G, Bjorner J, Brazier J, et al. Cross-validation of item selection and scoring for the SF-12 Health Survey in nine countries: Results from the IQOLA Project. J Clin Epidemiol 1998; 51: 1171-8. 
31. Ware JE, Kosinski M, Keller SD. A 12-item short form health survey. Med Care 1996; 34: 220-3.

32. Ministerio de Salud. II Encuesta de Calidad de Vida y Salud Chile, 2006. Subsecretaría de Salud Pública, División de Planificación Sanitaria. 2006. Diciembre. Disponible en http://epi.minsal.cl/epi/html/sdesalud/ calidaddevida2006/Informe\%20Final\%20Encuesta\%20 de\%20Calidad\%20de\%20Vida\%20y\%20Salud\%202006. pdf. [Consultado el 03 de enero de 2011].

33. Ashing-Giwa KT, Kim J, Tejero JS. Measuring quality of life among cervical cancer survivors: Preliminary assessment of instrumentation validity in a cross-cultural study. Qual Life Res 2008; 17, 147-57.

34. Failde I, Medina P, Ramírez C, Arana R. Construct and criterion validity of the SF-12 health questionnaire in patients with acute myocardial infarction and unstable angina. J Eval Clin Practice 2010; 16: 569-73.

35. Mols F, Pelle A, Kupper N. Normative data of the SF-12 health survey with validation using postmyocardial infarction patients in the dutch population. Qual Life Res 2009; 18: 403-14.

36. Zúñiga C, Dapueto J, Müller H, Kirsten L, Alid R, Ortiz L. Evaluación de la calidad de vida en pacientes en hemodiálisis crónica mediante el cuestionario Kidney Disease Quality of Life (KDQOL-36). Rev Med Chile 2009; 137 (2): 200-7.

37. Jakobsson U. Using the 12 -item short form health survey (SF-12) to measure quality of life among older people. Aging Clin Exp Res 2007; 19: 457-64.

38. Beck AT, Rush AJ, Shaw BF, Emery G. Cognitive therapy of depression. New York: Guilford Press 1979. (Traducido al español en Bilbao: Desclée de Brower, 1983).

39. Beltrán MC, Freire MA, Hernández L. El Inventario de Depresión de Beck: Su validez en población adolescente. Ter Psicol 2012; 30: 5-13.

40. Sanz J, Vásquez C. Fiabilidad, validez y datos normativos del inventario para la depresión de Beck. Psicothema 1998; 10 (2): 303-18.

41. Cruz-Sáez MS, Salaberria K, Rodríguez S, Echeburúa E. Imagen corporal y realización de dieta. Diferencias entre adolescentes españolas y latinoamericanas. Univ Psychol 2013; 12 (3): 699-708.

42. Melipillán-Araneda R, Cova-Solar F, Rincón-González P, Valdivia-Peralta M. Propiedades psicométricas del inventario de depresión de Beck-II en adolescentes chilenos. Ter Psicol 2008; 26 (1): 59-69.

43. González-Ramírez MT, Landero-Hernández R, GarcíaCampayo J. Relación entre la depresión, la ansiedad y los síntomas psicosomáticos en una muestra de estudiantes universitarios del norte de México. Rev Panam Salud Pública 2009; 25 (2): 141-5.
44. Spielberger Ch, Gorsuch R, Luschene R. Manual for the state-trait anxiety inventory. Palo Alto, CA: Consulting Psychologist Press, 1970. (Adapt. Española, TEA, 1982).

45. Bermúdez J. Anxiety and performance. Rev Psicol Gen Apl 1978a; 151: 183-207.

46. Bermúdez J. Functional analysis of anxiety. Rev Psicol Gen Apl 1978b; 153: 617-34.

47. Vera-Villarroel P, Celis-Atenas K, Córdova-Rubio N, Buela-Casal G, Spielberger CD. Preliminary analysis and normative data of the state-trait anxiety inventory (STAI) in adolescent and adults of Santiago, Chile. Ter Psicol 2007; 25 (2): 155-62.

48. Huppert F, Whittington J. Evidence for the independence of positive and negative well-being: Implications for quality of life. Br J Health Psychol 2003; 8: 107-22.

49. The WHOQOL Group. The World Health Organization Quality of life assessment (WHOQOL): Position paper from the world health organization. Social Science and Medicine 1995; 41: 1403-9.

50. Montazeri A, Vahdaninia M, Javad Mousavi S, Omidvari $\mathrm{S}$. The Iranian version of 12-item short form health survey (SF-12): Factor structure, internal consistency and construct validity. BMC Public Health 2008; 9: 341-51.

51. Okonkwo OC, Roth DL, Pulley LV, Howard G. Confirmatory factor analysis of the validity of the SF-12 for persons with and without a history of stroke. Qual Life Res 2010; 19: 1323-31.

52. Scientific Advisory Committee of the Medical Outcomes Trust. Assessing health status and quality-of-life instruments: Attributes and review criteria. Qual Life Res 2002; 11: 193-205.

53. Cohen RJ, Swerdlik ME. Pruebas y evaluación psicológicas. Introducción a las pruebas y a la medición. México: Mc Graw Hill. 1996.

54. Fleishman J, Selim A, Kazis L. Deriving SF-12v2 physical and mental health summary scores: A comparison of different scoring algorithms. Qual Life Res 2010; 19: 231-41.

55. Kodraliu G, Mosconi P, Groth N, Carmosino G, Perilli A, Gianicolo EA, et al. Subjective health status assessment: Evaluation of the Italian version of the SF-12 Health Survey. Results from the MiOS Project. J Epidemiol Biostat 2001; 6: 305-16.

56. Kontodimopoulos N, Pappa E, Niakas D, Tountas Y. Validity of SF-12 summary scores in a Greek general population. Health Qual Life Outcomes 2007; 5: 55-64.

57. Urzúa A, Bravo M, Ogalde M, Vargas C. Factores vinculados a la calidad de vida en la adultez mayor. Rev Med Chile 2011; 139 (8): 1006-14.

58. Kerlinger F, Lee H. Investigación del comportamiento. Métodos de investigación en ciencias sociales. México: Mc 
Evaluación del cuestionario SF-12 - P. Vera-Villarroel et al

Graw Hill 2002.

59. Antúnez Z, Vinet E. Escalas de depresión, ansiedad y Estrés (dass-21): Validación de la Versión abreviada en Estudiantes Universitarios Chilenos. Ter Psicol 2012; 30 (3): 49-55.

60. Cova F, Rincón P, Inostroza C, Melipillán R. Factores asociados prospectivamente a sintomatología depresiva en adolescentes tempranos de sexo femenino. Univ Psychol 2012; 11 (3): 853-62.

61. Lillo S, Martini N. Principales tendencias iberoamericanas en psicología clínica. Un estudio basado en la evidencia. Ter Psicol 2013; 31 (3): 363-71. 